\title{
An enriched constitutive modelling framework for localised failure of geomaterials
}

\section{Marco para un modelo constitutivo extendido para geomateriales con fallas localizadas}

\section{Giang D. Nguyen}

Fecha de entrega: 6 de diciembre 2013 Fecha de aceptación: 5 de mayo 2014

School of Civil, Environmental and Mining Engineering, North Terrace Campus, The University of Adelaide, SA 5005 Australia, g.nguyen@adelaide.edu.au

Localised failure in geomaterials is preceded and accompanied by intensive deformation and irreversible micro-structural changes of the material in a small but finite size region. Shear, compaction, and dilation bands observed in soils and porous rocks are typical examples of phenomena that lead to localised failure. The width $h$ of the localisation band has been experimentally shown to be a physical quantity related to the microstructure of the material. On the other hand, numerical methods for the solution of boundary value problems usually introduce another length scale $H$, as a result of the spatial discretisation of the considered domain into smaller ones over which the constitutive response of the material is defined in terms of incremental stressstrain relationships. While $h$, as a physical quantity, is fixed, $H$ varies with the resolution of the numerical discretisation. Since $h$ scales with the material microstructure and therefore is usually much smaller than the resolution of the numerical discretisation, the case $H>h$ is considered in this study, e.g. failure behaviour governed by a localisation band of width $h$ embedded in an elastic bulk of nominal side H. We present a general constitutive modelling framework to connect these two scales, and corresponding responses of the materials inside and outside the localisation zone. We demonstrate how this approach can help obtain physically meaningful solutions that are independent of the spatial discretisation in numerical analysis. Numerical analyses of localised failure in quasi-brittle materials are used to further highlight the features and applicability of the proposed approach.

Keywords: length scales, constitutive modelling, localised failure, discontinuity, bifurcation, damage, fracture energy
Fallas localizadas en geomateriales están precedidas y se dan en conjunto con deformaciones intensas y cambios micro estructurales irreversibles del material en una región de tamaño finito, pero pequeña. Corte, compactación y bandas de dilatación observables en suelos y rocas porosas son ejemplos típicos de fenómenos que conducen a fallas localizadas. Ha sido experimentalmente demostrado que el ancho $h$ de la banda de localización es una cantidad física relacionada con la micro estructura del material. Por otro lado, métodos numéricos para la resolución de problemas de valor en la frontera usualmente introducen otra longitud de escala H como un resultado de la discretización espacial del dominio considerado en partes más pequeñas sobre el cual la respuesta constitutiva del material está definida en términos de relaciones incrementales de tensión-deformación. Mientras $h$ como cantidad fisica está fija, $H$ varía con la resolución de la discretización numérica. Dado que $h$ escala con la micro estructura del material y por lo tanto es usualmente mucho más pequeño que la resolución de la discretización numérica, el caso $H>$ h es considerado en este estudio, por ejemplo el comportamiento en falla gobernado por una banda de localización de ancho h inserta en una masa elástica de lado nominal H. Se presenta un marco de modelamiento constitutivo general para conectar estas dos escalas, y las respuestas correspondientes del material dentro y fuera de la zona de localización. Se demuestra como esta estrategia puede ayudar a obtener soluciones con significado fisico que son independientes de la discretización espacial en análisis numéricos. Análisis numéricos de falla localizada en materiales cuasi frágiles son además usados para destacar las características y aplicabilidad de la estrategia propuesta. Palabras clave: escalas de longitud, modelo constitutivo, falla localizada, discontinuidad, bifurcación, daño, energía de fracturación 


\section{Introduction}

Failure of geomaterials such as the formation of sea ice leads in the Arctic (Jirasek and Bazant, 1995; Sulsky et al., 2007), rock fracture in underground mining, etc., usually involves the material behaviour at various scales and stages of deformation. In particular, inelastic deformation and fracture localise in narrow zones, while the surrounding bulk, usually of several orders of magnitude larger in extent, is unloading elastically. Numerical modelling of such large scale failure processes (dimensions of several kilometres) is computationally challenging, as the behaviour of the material inside and outside the localisation zone should be incorporated in a numerical model. In this regard, classical continuum models usually lack a length scale to correctly capture the localised failure of softening materials. While enrichment of such models with an internal length through the use of nonlocal/gradient regularisation (PijaudierCabot and Bazant, 1987; Chen and Schreyer, 1987) is a mathematically and probably physically rigorous way, the application of such enhancements is severely restricted by the available computational resources. This is because the locations of the failure zones are generally unknown and the considered domain can be of several orders of magnitude bigger than the characteristic width $h$ of the localisation zone, resulting in a very high resolution of the discretisation, and consequently very large model size.

In the literature, fracture energy regularisation based on the smeared crack concept (Cedolin and Bazant, 1980) is probably the simplest way to cope with softening-related issues in the failure analysis of solids/structures. However, this suffers from the drawback that the constitutive behaviour must be unphysically scaled with the resolution of the discretisation to meet the requirement on the energy dissipation. In addition, once a coarse spatial discretisation is used (e.g. in large scale analysis), the specific fracture energy obtained from the (surface) fracture energy and the resolution of the discretisation (see equation (8) below) becomes smaller than the elastic strain energy at peak (Jirasek and Bazant, 1995), resulting in the inadmissible snapback instability in the constitutive response. Other enhancements to the discretisation scheme include Enhanced Assumed Strain EAS (e.g. Larsson et al., 1996; Oliver, 1996; Borja, 2000; Foster et al., 2007) and XFEM, the eXtended Finite Element Methods (e.g. Wells and
Sluys, 2001; Samaniego and Belytschko, 2005; Sanborn and Prévost, 2011). These more sophisticated methods have been extensively used to address failure modelling at large scales, e.g. $H>h$, and usually idealise the finite width localisation zone as a zero thickness surface across which the displacement field is discontinuous (the strong discontinuity case). We are also aware of earlier works on weak discontinuity (e.g. only the strain field is discontinuous) using the localisation zone embedded in finite elements (e.g. Belytschko et al., 1988; Sluys and Berends, 1998; and Garikipati and Hughes, 2000). The key idea of these approaches is to enhance the deformation mode of the special finite element so that both inelastic behaviour in the localisation zone and the elastic shrinking of the bulk continuum can be adequately accounted for. As a consequence, all such approaches involve finite element re-formulations, e.g. modification and/or introduction of shape functions, and hence result in the dependence of the approach on the type of finite element used for the numerical discretisation. Can we come up with a methodology better than the smeared crack approach, but less complex than the EAS or XFEM?

The key idea described in the present paper is to enhance the constitutive behaviour description, rather than the finite element formulation, with a length scale related to the width of the localisation zone used for large scale analysis of failure, e.g. $h<H$. We address the nature of the localised failure from the constitutive modelling point of view without having to resort to variational formulations for the discretisation using the finite element method. The interface with any spatial discretisation scheme is taken into account only through the size $h$ of the sub-domain (Figure 1), while connection with any kind of constitutive behaviour is specified through its tangent stiffness. The proposed approach is therefore straightforwardly applicable to any material model and any spatial discretisation scheme.
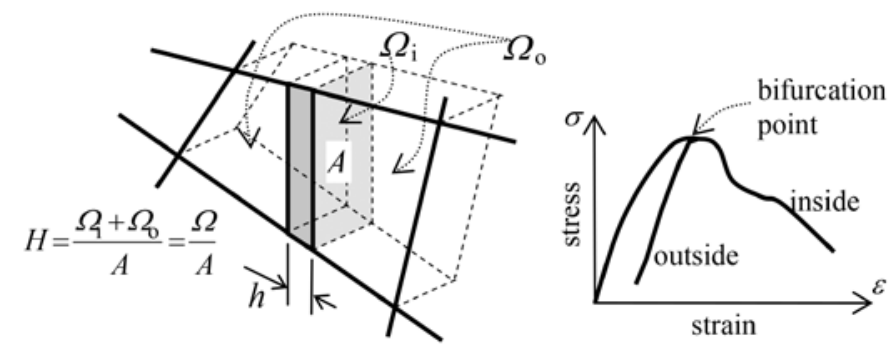

Figure 1: Localisation zone (shaded) embedded in a volume (Nguyen et al., 2012) 


\section{Constitutive modelling framework}

We aim to develop at first a general framework that can fit any constitutive model. The starting point is the localisation zone of size $h$ embedded in a volume of nominal size $H$, introduced in relation to the Fracture Process Zone FPZ observed in the failure of quasi-brittle materials, or the shear band in soils. This is the stage beyond the homogeneous deformation of the material (Figure 1), e.g. at the onset or after the bifurcation of the material behaviour (Borja, 2000). It is assumed in this framework that, during further deformation, the material undergoes elastic unloading in the region outside the localisation zone. While $h$ is related to the inelastic behaviour and failure at the lower scale, the size $H$ of the elastic bulk is merely a numerical feature resulting from the discretisation of the domain under consideration. Grid spacing in the finite difference method and the domain size ascribed to an integration point in the finite element method are typical examples of the meaning we attach to the discretisation characteristic size $H$. As a consequence, $H$ can vary depending on the required resolution of the numerical discretisation, while $h$ can be considered a material property and hence is invariant with the numerical discretisation. We view the configuration at failure in Figure 1 as a composite material consisting of two phases: an inelastic localisation zone embedded in an elastic bulk. For this, the linear scaling of the total strain rate applies:

$$
\dot{\boldsymbol{\varepsilon}}=f \dot{\boldsymbol{\varepsilon}}_{\mathrm{i}}+(1-f) \dot{\boldsymbol{\varepsilon}}_{\mathrm{o}}
$$

where $f=h / H$ is the volume fraction of the embedded localisation zone in $\Omega$ and subscripts " $i$ " and "o" are used to denote quantities belonging to the behaviour inside and outside the localisation zone, respectively (Figure 1). Across the boundary of the localisation zone, the internal equilibrium in terms of traction continuity must be met, enforcing coupling between the inelastic localisation zone and outside elastic bulk. In other words, the elastic bulk is enhanced by the inelastic behaviour of the localisation zone (Nguyen et al., 2012). Denoting $\mathbf{n}$ the normal vector, [̊] the relative velocity between opposite sides of the localisation band, and adopting the following form for the strain rate $\dot{\varepsilon}_{\mathrm{i}}$ inside the localisation band (Vardoulakis et al., 1978; Kolymbas, 2009; neglecting the small homogeneous term in $\dot{\boldsymbol{\varepsilon}}_{\mathrm{i}}$ ):

$$
\dot{\boldsymbol{\varepsilon}}_{\mathrm{i}}=\frac{1}{h}(\mathbf{n} \otimes[\dot{\mathbf{u}}])^{s}=\frac{1}{2 h}(\mathbf{n} \otimes[\dot{\mathbf{u}}]+[\dot{\mathbf{u}}] \otimes \mathbf{n})
$$

the relaxation strain rate $\dot{\varepsilon}_{\mathrm{o}}$ of the elastic bulk is:

$$
\begin{aligned}
\dot{\boldsymbol{\varepsilon}}_{\mathrm{o}} & =\frac{1}{1-f}\left(\dot{\boldsymbol{\varepsilon}}-f \dot{\boldsymbol{\varepsilon}}_{\mathrm{i}}\right)=\frac{1}{1-f}\left[\dot{\boldsymbol{\varepsilon}}-f \frac{1}{h}(\mathbf{n} \otimes[\dot{\mathbf{u}}])^{s}\right] \\
& =\frac{1}{1-f}\left[\dot{\boldsymbol{\varepsilon}}-\frac{1}{H}(\mathbf{n} \otimes[\dot{\mathbf{u}}])^{s}\right]
\end{aligned}
$$

For elastic unloading of the bulk material with stiffness modulus $\mathbf{a}_{\mathbf{0}}$, the stress rate $\dot{\boldsymbol{\sigma}}$ of the continuum model is (Nguyen et al., 2012):

$$
\dot{\boldsymbol{\sigma}}=\dot{\boldsymbol{\sigma}}_{\mathrm{o}}=\mathbf{a}_{\mathrm{o}}: \dot{\boldsymbol{\varepsilon}}_{\mathrm{o}}=\frac{1}{1-f} \mathbf{a}_{\mathrm{o}}:\left(\dot{\boldsymbol{\varepsilon}}-f \dot{\boldsymbol{\varepsilon}}_{\mathrm{i}}\right)
$$

Inside the localisation zone, the constitutive relationship rate is assumed of the general form:

$$
\dot{\boldsymbol{\sigma}}_{\mathrm{i}}=\mathbf{a}_{\mathrm{i}}: \dot{\varepsilon}_{\mathrm{i}}
$$

where $\mathbf{a}_{\mathrm{i}}$ is the tangent stiffness. The above two equations are linked through the internal equilibrium dictating the continuity of traction across the boundary of the localisation zone:

$$
\left(\dot{\boldsymbol{\sigma}}-\dot{\boldsymbol{\sigma}}_{\mathrm{i}}\right) \cdot \mathbf{n}=0
$$

The distinction from a regular constitutive model lies in the coupled equations (4-6). From (4), in order to obtain the stress rate $\dot{\boldsymbol{\sigma}}$ from a given strain rate $\dot{\boldsymbol{\varepsilon}}$, the constitutive equation (5) dictating the inelastic response of the localisation band, together with the internal equilibrium equation (6), are needed. The volume fraction $f$ and the inelastic response inside the localisation zone contribute to the relaxation of the stress rate in the elastic bulk. Equations (4-5) can be worked out to result in the following form of stress-strain relationship (Nguyen et al., 2012):

$$
\dot{\boldsymbol{\sigma}}=\frac{1}{1-f} \mathbf{a}_{\mathrm{o}}:\left[\dot{\boldsymbol{\varepsilon}}-\frac{1}{H}\left(\mathbf{n} \otimes\left(\mathbf{C}^{-1} \cdot\left(\mathbf{a}_{\mathrm{o}}: \dot{\boldsymbol{\varepsilon}}\right) \cdot \mathbf{n}\right)\right)^{s}\right]
$$
$\mathrm{H} / \mathrm{h}=2$ 
zones, in addition to the anisotropy introduced by the localisation. Size effects are therefore explicitly integrated in the constitutive behaviour.

\section{What are the new features?}

The above approach can be viewed as a two-stage smearing process. The velocity jump $[\dot{\mathbf{u}}]$ between the two sides of the localisation band is at first smeared over the localisation band with physical size $h$ (equation (2)). Therefore the integration of inelastic constitutive behaviour for stress and internal variables in the localisation zone is only related to this physical size and are invariant with respect to the discretisation size $H$. To obtain the relaxation strain rate and then stress rate of the elastic bulk, a compatibility argument (equation (3)) is utilised, effectively consisting of further smearing of the velocity jump $[\dot{\mathbf{u}}]$ over the discretisation size $H$. The difference with the traditional smeared crack approach is obvious: in the latter, everything is smeared over the discretisation size $H$, meaning that the constitutive response must vary with the resolution of the discretisation to maintain the same amount of energy dissipation. As a consequence, very big $H$ required in large scale modelling due to limited computing resource leads to unphysical scaling of model parameters which may still be unable to prevent snapback in the constitutive behaviour (e.g. Jirasek and Bazant, 1995; Sulsky et al., 2007).

The current approach, in contrast, gives a direct access to additional degrees of freedom related to the strain within the localisation band of size $h$, in which the constitutive law is physical and hence does not snap back. Numerically, this means that the stress return algorithms for rate constitutive equations can always be performed.

We illustrate the above points using a simple constitutive model in 1D setting (Figure 2). This is a softening behaviour observed in failure of quasibrittle materials like rocks or concrete in which the pre- and post-peak responses are represented by the slopes $a_{\mathrm{o}}$ and $a_{\mathrm{i}}$ of the stress-strain curve. The material is homogeneous up to the peak point, after which the response bifurcates into two branches with slopes $a_{\mathrm{i}}$ and $a_{\mathrm{o}}$ corresponding to the inelastic behaviour inside the Fracture Process Zone FPZ of size $h$ and elastic behaviour in the outside bulk of size $H-h$ (Figure 2).
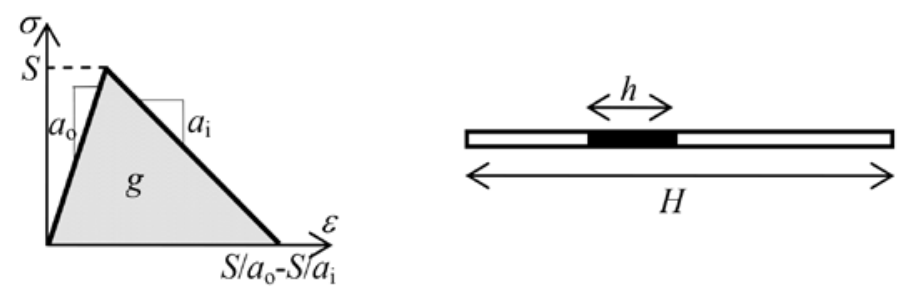

Figure 2: 1D constitutive behaviour and corresponding sizes

The relationship between the FPZ size $h$, specific dissipation $g$, as the area under the stress-strain curve, and fracture energy $G$, as the energy released due to the creation of new surface area can be written as:

$$
G=h g=\frac{h S^{2}}{2} \frac{a_{\mathrm{i}}-a_{\mathrm{o}}}{a_{\mathrm{i}} a_{\mathrm{o}}}
$$

The internal equilibrium in this $1 \mathrm{D}$ case reads: $\dot{\sigma}_{\mathrm{o}}=\dot{\sigma}_{\mathrm{i}}$ while the strain rate inside the FPZ takes the simple form $\dot{\varepsilon}_{\mathrm{i}}=[\dot{u}] / h$. From equations (4-6) we can write:

$$
\frac{1}{1-f} a_{\mathrm{o}}\left(\dot{\varepsilon}-f \dot{\varepsilon}_{\mathrm{i}}\right)=a_{\mathrm{i}} \dot{\varepsilon}_{\mathrm{i}}
$$

This results in the strain rate $\dot{\varepsilon}_{\mathrm{i}}$ inside the localisation zone in terms of the total strain rate $\dot{\varepsilon}$, as:

$$
\dot{\varepsilon}_{\mathrm{i}}=\frac{a_{\mathrm{o}} \dot{\varepsilon}}{(1-f) a_{\mathrm{i}}+f a_{\mathrm{o}}}
$$

Using (4), we can then write the stress rate $\dot{\sigma}$ in terms of strain rate $\dot{\varepsilon}$, as:

$$
\dot{\sigma}=\frac{1}{1-f} a_{\mathrm{o}}\left(\dot{\varepsilon}-f \dot{\varepsilon}_{\mathrm{i}}\right)=\frac{a_{\mathrm{i}} a_{\mathrm{o}} \dot{\varepsilon}}{(1-f) a_{\mathrm{i}}+f a_{\mathrm{o}}}
$$

In the context of quasibrittle failure, the fracture energy $G$ (equation (8)) is a material constant representing the contributions from the release of surface energy due to micro-cracking in the FPZ of size $h$. Therefore the inelastic behaviour in the localisation zone characterised by the softening modulus $a_{\mathrm{i}}$ (Figure 2) is dependent on $G$ and the size $h$ of the FPZ, as intrinsic material parameters. Expressing everything in terms of $G$ and $h$, we obtain:

$\dot{\varepsilon}_{\mathrm{i}}=\dot{\varepsilon}\left(1-\frac{2 G a_{\mathrm{o}}}{h S^{2}}\right) /\left(1-\frac{2 G a_{\mathrm{o}}}{H S^{2}}\right)$ 


$$
\dot{\sigma}=a_{\mathrm{o}} \dot{\varepsilon} /\left(1-\frac{2 G a_{\mathrm{o}}}{H S^{2}}\right)
$$

The stress rate in (13) is exactly that obtained from a smeared crack approach, e.g. smearing $G$ over the size $H$ of the finite element. Snapback in the overall response, e.g. $\dot{\sigma} / \dot{\varepsilon}>0$ and $\dot{\sigma}<0$, is present if $H$ is sufficiently large to make the denominator positive. However, the constitutive response is integrable thanks to the use of an internal equilibrium equation (6), together with the enrichment stress $\sigma_{i}$ and strain $\varepsilon_{i}$. Regardless of the discretisation size $H$, the strain rate $\dot{\varepsilon}_{\mathrm{i}}$ of the response inside the FPZ is always a positive quantity representing the separation of the material. Therefore beyond the elastic regime, a negative value of $\dot{\varepsilon}$, due to snapback in the overall response, still results in a positive strain rate $\dot{\varepsilon}_{i}$ for the integration of inelastic incremental constitutive equations. In the context of numerical failure analysis, $\varepsilon_{i}$ is monotonically increasing with failure progression and it can be used as a control parameter in any indirect displacement control solution schemes (e.g. the local arc-length control by May and Duan (1997) and Yang and Proverbs, (2004)).

Physically, snapback in a structural sense (e.g. finite element response) is a consequence of insufficient resolution of the discretisation in large scale failure analysis that, according to the literature, usually requires the enhancement of deformation mode of finite elements, e.g. using EAS or XFEM type enrichments. The proposed framework is another kind of enhancement, relying solely on the constitutive behaviour at integration point level with the use of the enrichment stress $\sigma_{i}$ and strain $\varepsilon_{i}$. The advantage over traditional smeared crack is obvious, while the simplicity and practicality advantages over sophisticated enrichment methods like EAS or XFEM can also be seen. We will further illustrate this point in a forthcoming paper.

\section{Numerical examples}

The first numerical example shows the response of a bar of length $H$, with unit cross sectional area and an embedded FPZ of size $h$ (see Figure 2). Only the constitutive response is concerned in this example, which is equivalent to the behaviour of a single linear 1D finite element of length $H$. The fracture energy is taken as 15 times the elastic strain energy at peak. Figure $3 \mathrm{~b}$ shows the effects of varying the bar length $H$ on the overall response, while keeping $h$ fixed. It can be seen that regardless of the response, the area under the stress-displacement curve remains unchanged and is always equal to the dissipation in the FPZ (see equation (8)). The stress strain response inside the FPZ (Figure 3a) is invariant with the size $H$, while that is not the case with smeared crack approach (Figure 3c) due to the fact that everything is smeared over the bar length $H$. In short, the single stress component in the smeared crack approach requires the variation of the constitutive behaviour with respect to the discretisation to meet the requirement on the dissipation. The stress-strain behaviour in such cases just reflects the overall stress-displacement response, e.g. essentially strain is obtained by smearing the displacement over the length $H$. The coupled stresses in the proposed constitutive modelling framework allow us to keep a meaningful constitutive response inside the FPZ that is invariant with the discretisation (Figure 3a). Essentially, all parameters of the model remain unchanged with respect to the resolution of the discretisation, a property that is missing in traditional smeared crack approach.
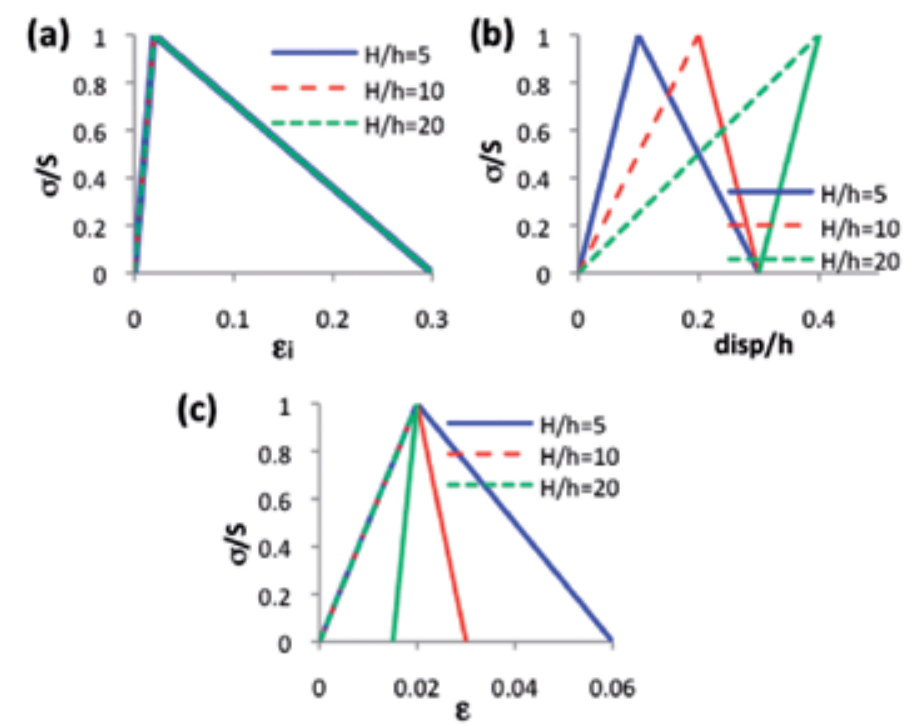

Figure 3: Size effects on constitutive behaviour: a) normalised stress-strain response in the FPZ, b) normalised stress-normalised displacement, and c) normalised stress-strain response of a smeared crack model

In the second example, a $10 \mathrm{~mm}$ long bar, clamped at one end and free at the other, is discretised using 2D finite elements under plane strain conditions. Localised failure is triggered off by weakening the element next to the clamp (tensile strength reduced by $10 \%$ ). We used a linear softening law (see expressions (8-13)) with the following 
properties: Young's modulus $E=30000 \mathrm{MPa}$, Poisson's ratio $v=0.2$, tensile uniaxial strength $S=3 \mathrm{MPa}$, and fracture energy $\mathrm{G}=0.0015 \mathrm{Nmm} / \mathrm{mm}^{2}$. Figure 4a shows that the structural response of the bar is insensitive to the discretisation. The evolution of the displacement profiles in 2 cases, coarse and fine meshes, are also depicted and seen to coincide above the resolution of the coarse mesh.

In the third example, a three point bending test is analysed using a simplified version of the constitutive model proposed in Nguyen and Korsunsky (2008), embedded in the above constitutive modelling scheme. The model is implemented in an in-house numerical code based on the Material Point Method MPM (Sulsky et al., 1995). Details on the implementation of the model along with some computational issues related to the application of the proposed scheme will be covered in a forthcoming paper. The geometrical data and material properties are taken from the experimental test of Petersson (1981) and illustrated in Figure 5. As can be seen in Figure 5, the numerical responses are insensitive to the resolution of the discretisation and also the integration scheme of the MPM. Integration schemes using 4 and 16 material points MPs per element result in almost same response. In all cases, the numerical predictions closely follow the experimental trends.
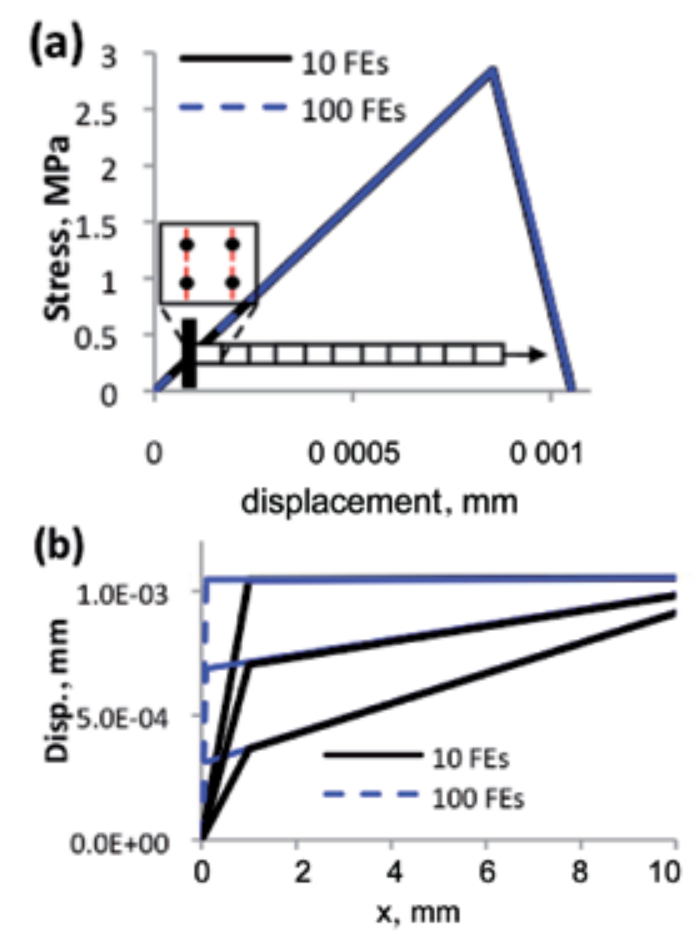

Figure 4: A bar under tension: a) load-displacement response, and b) displacement profiles
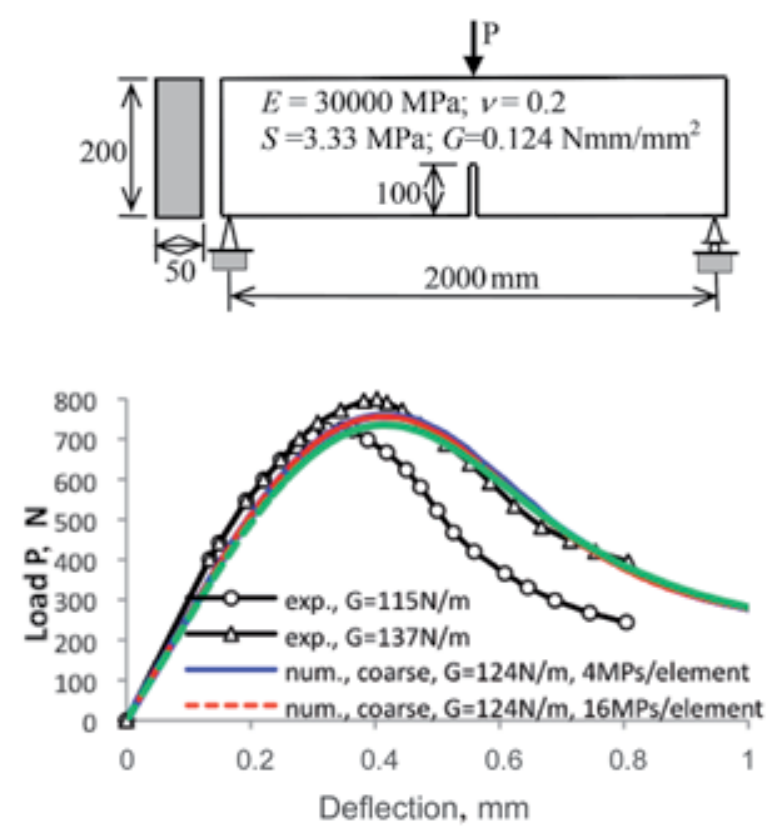

Figure 5: Three-point bending test: geometry and load-deflection response

\section{Conclusions}

We developed a new framework that allows the integration of a length scale in constitutive models. The development is based on the localised nature of failure in geomaterials and utilises the internal equilibrium across the localisation band. This results in constitutive models possessing a length scale, and featuring coupled stress behaviour. The fact that both kinematical compatibility and traction continuity are enforced at the constitutive level in the formulation, e.g. at integration points, makes the implementation in any numerical code straightforward. As a consequence, the new approach is applicable to any existing constitutive model and also any discretisation scheme. This is totally different from traditional approaches that always require the unphysical scaling of model parameters with the resolution of the discretisation (e.g. smeared crack approach), or modification of existing finite elements (e.g. EAS or XFEM). Numerical examples in this paper, in the context of quasibrittle failure, demonstrate the essential features and the promising performance of the new approach.

\section{Acknowledgements}

Financial support from the Australian Research Council for project DP1093485 is gratefully acknowledged. 


\section{References}

Belytschko, T., Fish, J. and Engelmann, B.E. (1988). A finite element with embedded localization zones. Computer Methods in Applied Mechanics and Engineering 70, 59-89

Borja, R.I. (2000). A finite element model for strain localization analysis of strongly discontinuous fields based on standard Galerkin approximation. Computer Methods in Applied Mechanics and Engineering 190, 1529-1549

Cedolin L. and Bazant, Z.P. (1980). Effect of finite element choice in blunt crack band analysis. Computer Methods in Applied Mechanics and Engineering 24(3):305-316

Chen, Z. and Schreyer, H.L. (1987). Simulation of soil-concrete interfaces with nonlocal constitutive models. Journal of Engineering Mechanics 113, 1665-1677

Foster, C.D., Borja, R.I. and Regueiro, R.A. (2007). Embedded strong discontinuity finite elements for fractured geomaterials with variable friction. International Journal for Numerical Methods in Engineering 72, 549-581

Garikipati, K. and Hughes, T.J.R. (2000). A variational multiscale approach to strain localization - formulation for multidimensional problems. Computer Methods in Applied Mechanics and Engineering 188(1-3), 39-60

Jirasek, M. and Bazant, Z.P. (1995). Particle model for quasibrittle fracture and application to sea ice. Journal of Engineering Mechanics 121, 1016-1025

Kolymbas, D. (2009). Kinematics of shear bands. Acta Geotechnica 4, 315-318

Larsson, R., Runesson, K. and Sture, S. (1996). Embedded localization band in undrained soil based on regularized strong discontinuity - theory and FE-analysis. International Journal of Solids and Structures 33, 3081-3101

May, I.M. and Duan, Y. (1997). A local arc-length procedure for strain softening. Computers \& Structures 64(1-4), 297-303

Nguyen, G.D., Einav, I. and Korsunsky, A.M. (2012). How to connect two scales of behaviour in constitutive modelling of geomaterials. Géotechnique Letters (special issue on Geomechanics Across the Scales) 2(3), 129-134

Nguyen, G.D. and Korsunsky, A.M. (2008). Development of an approach to constitutive modelling of concrete: isotropic damage coupled with plasticity. International Journal of Solids and Structures 45(20), 5483-5501
Oliver, J. (1996). Modelling strong discontinuities in solid mechanics via strain softening constitutive equations. Part 1: fundamentals. International Journal for Numerical Methods in Engineering 39, 3575-3600

Petersson, P.E. (1981). Crack growth and development of fracture zones in plain concrete and similar materials. Report TVBM-1006, Div. of Build. Mat., Lund Institute of Technology, Lund, Sweden

Pijaudier-Cabot, G. and Bazant, Z.P. (1987). Nonlocal damage theory. Journal of Engineering Mechanics 113(10), 1512-1533

Samaniego, E. and Belytschko, T. (2005). Continuumdiscontinuum modelling of shear bands. International Journal for Numerical Methods in Engineering 62, 1857-1872

Sanborn, S.E. and Prévost, J.H. (2011). Frictional slip plane growth by localization detection and the extended finite element method (XFEM). International Journal for Numerical and Analytical Methods in Geomechanics 35, 1278-1298

Sluys, L.J. and Berends, A.H. (1998). Discontinuous failure analysis for mode-I and mode-II localization problems. International Journal of Solids and Structures 35, 4257-4274

Sulsky D., Zhou S-J. and Schreyer, H.L. (1995). Application of a particle-in-cell method to solid mechanics. Computer Physics Communications 87, 236-252

Sulsky, D., Schreyer, H., Peterson, K., Kwok, R. and Coon, M. (2007). Using the material-point method to model sea ice dynamics. Journal of Geophysical Research 112, C02S90

Vardoulakis, I., Goldscheider, M. and Gudehus, G. (1978). Formation of shear bands in sand bodies as a bifurcation problem. International Journal for Numerical and Analytical Methods in Geomechanics 2, 99-128 Wells, G.N. and Sluys, L.J. (2001). A new method for modelling cohesive cracks using finite elements. International Journal for Numerical Methods in Engineering 50, 2667-2682

Yang, Z.J. and Proverbs, D. (2004). A comparative study of numerical solutions to non-linear discrete crack modeling of concrete beams involving sharp snap-back. Engineering Fracture Mechanics 71, 81-105 